\title{
CONTINUOUS-DOMAIN ARMA MODELING FOR ULTRASOUND TISSUE CHARACTERIZATION
}

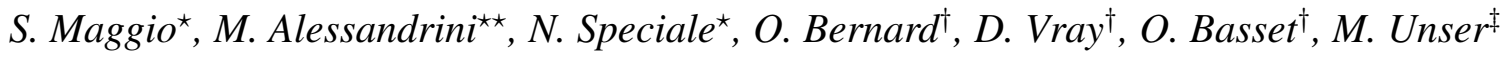 \\ ${ }^{\star}$ Department of Electronics, Computer Sciences and Systems, Università di Bologna, Italy \\ ${ }^{\star \star}$ ARCES, Università di Bologna, Bologna, Italy \\ ${ }^{\dagger}$ CREATIS, CNRS UMR5220, INSERM U630, Université de Lyon, Insa-Lyon, Villeurbanne, France \\ ${ }^{\ddagger}$ Biomedical Imaging Group, École polytechnique fédérale de Lausanne, Switzerland
}

\begin{abstract}
In this study, we investigate the possibility of applying a continuous-time ARMA (CARMA) model to radio-frequency ultrasound signals. We consider the effect of the discretization process on the parameters of the continuous system, and we take into account the exponential nature of the autocorrelation function of the model to derive continuous-domain information from the parameters of the discrete ARMA process. We validate the effectiveness of the CARMA model parameters for the characterization of ultrasound tissues on a sequence of phantom images that represent various concentrations of scatterers. We also compare the proposed CARMA coefficients and the traditional ARMA parameters on the basis of their performance in discriminating between phantom tissues. We show that working in the continuous domain brings additional useful information to characterize the imaged materials.
\end{abstract}

Index Terms - ARMA, CARMA, ultrasound, tissue characterization, exponential spline

\section{INTRODUCTION}

In ultrasound diagnostic, the acquired image is a rich source of information pertaining to the imaged tissue, even though some of it is not directly accessible for human visual inspection. To reveal this hidden information, it is necessary to resort to computer-aided tissue characterization to capture several features carried by medical sonography and to correlate them to the state of the imaged tissue. In particular, the statistical modeling of discrete ultrasound signals has been widely employed to derive interesting properties from estimated model parameters that are useful in the context of tissue characterization. Among them, shift-variant autoregressive moving-average (ARMA) processes were found suitable to model the ultrasound signals and images and to make the acquisition more robust in its estimation of the uncorrupted tissue response [1,2]. More recently, 2D ARMA modeling was proposed to improve computer-aided detection of breast tumors $[3,4]$.
Although continuous-time ARMA (CARMA) stochastic processes have been successfully exploited for image modeling $[5,6]$, the continuous-domain approach has never been investigated in the processing of ultrasound images. In this study, we consider the radio-frequency (RF) ultrasound signal as a sampled CARMA process and recover its CARMA parameters from the traditional ARMA coefficients through an indirect approach.

The ARMA coefficients are easily computed by minimizing the prediction error [7]. Then, the CARMA parameters can be estimated from their discrete-domain counterpart by mapping the ARMA model back in the continuous domain [8]. In doing so, both the exact discretization of CARMA model and the exponential properties of the autocorrelation function in the discrete and continuous domains must be taken into account. As was shown in [9], the exact link between ARMA and its corresponding CARMA model can be established, provided one is willing to interpolate the discrete signal by exponential B-splines specific to the considered model [10,11]. In situations where a sufficiently high sampling frequency is employed, it is beneficial to exploit the dependence of the exponential B-spline on the ARMA parameters of the sampled model. This, along with a numerical optimization scheme, allows for the accurate estimation of the continuous-domain model parameters.

Here, we analyze the CARMA parameters of ultrasound signals in terms of their ability to capture information about the concentration of scatterers in imaged materials. For this purpose, we have created ultrasound images of phantoms with various concentrations of scatterers. The validation of CARMA and ARMA models in terms of discrimination performance shows that our continuous-based approach is more efficient than the traditional one. We believe that our unconventional ultrasound modeling is robust with respect to the effects of the system and has a good potential for tissue characterization.

We present the CARMA model for ultrasound signals in Section 2, and describe the indirect method for estimation of CARMA parameters in Section 3. The results of the com- 
parative analysis between CARMA and ARMA coefficients, performed on phantom images, are collected in Section 4. We conclude and discuss future perspectives on CARMA model for ultrasound images in Section 5.

\section{CARMA AND ARMA MODELING}

We assume that the spatial dependence of the effects of the acquisition system is weak. This allows us to focus on a segment of the ultrasound image and to consider that its characteristics are locally shift-invariant [12]. Then, the 1D discrete-time ultrasound signal $y[k]$ can be modeled as a spatially invariant sampled CARMA process $y(t)$, where the continuous-time Gaussian white noise $w(t)$ with variance $\sigma^{2}$ represents the innovation signal.

For a CARMA model of order $(n, m)$, the spectrum of $y(t)$ is expressed as

$$
\begin{aligned}
\Phi(\mathrm{j} \omega ; \boldsymbol{\pi}) & =\sigma^{2}\left|\frac{\prod_{i=1}^{m}\left(\mathrm{j} \omega-\beta_{i}\right)}{\prod_{i=1}^{n}\left(\mathrm{j} \omega-\alpha_{i}\right)}\right|^{2} \\
& =\sigma^{2}\left|\frac{\sum_{i=0}^{m} b_{i}(\mathrm{j} \omega)^{i}}{\sum_{i=0}^{n} a_{i}(\mathrm{j} \omega)^{i}}\right|^{2},
\end{aligned}
$$

where $\boldsymbol{\pi}=(\boldsymbol{\alpha}, \boldsymbol{\beta})=\left(\alpha_{1}, \ldots, \alpha_{n}, \beta_{1}, \ldots, \beta_{m}\right)$ is the collection of parameters that contains the poles $\alpha_{i}$ and the zeroes $\beta_{i}$ of the system, while $a_{i}$ and $b_{i}$ are the related polynomial coefficients.

In [9], it was shown that the sampling of the model (1) leads to a discrete ARMA model of order $(n, n-1)$. For the sake of simplicity, in the sequel we consider a unit-sampling interval. The discrete spectrum is then given by

$$
\Phi_{\mathrm{d}}(z ; \boldsymbol{\pi})=\lambda^{2} H_{\mathrm{d}}(z ; \boldsymbol{\pi}) H_{\mathrm{d}}\left(z^{-1} ; \boldsymbol{\pi}\right),
$$

where

$$
H_{\mathrm{d}}(z ; \boldsymbol{\pi})=\frac{\prod_{i=1}^{n-1}\left(1-\delta_{i} z^{-1}\right)}{\prod_{i=1}^{n}\left(1-\gamma_{i} z^{-1}\right)}=\frac{\sum_{i=0}^{n-1} d_{i} z^{-i}}{\sum_{i=0}^{n} c_{i} z^{-i}} .
$$

The sampled ultrasound signal, which represents the available data, is thus modeled as a discrete ARMA $(n, n-1)$. In this context the autocorrelation function of (1) is derived from its discrete samples thanks to interpolation by shifts of the exponential B-spline function $\eta_{(-\boldsymbol{\pi}: \boldsymbol{\pi})}(t)$ [10], where the symbol ":" indicates the union with additional opposite poles and zeroes, and where

$$
\eta_{\boldsymbol{\pi}}(t)=\mathscr{F}^{-1}\left\{\prod_{i=1}^{n} \frac{1-\mathrm{e}^{\alpha_{i}-\mathrm{j} \omega}}{\mathrm{j} \omega-\alpha_{i}} \prod_{i=1}^{m}\left(\mathrm{j} \omega-\beta_{i}\right)\right\}(t) .
$$

For this reason, the zeroes of the ARMA filter in (3) are the roots of the $z$-transform of the exponential B-spline samples $B_{(-\boldsymbol{\pi}: \boldsymbol{\pi})}(z)=\sum_{k \in \mathbb{Z}} \eta_{(-\boldsymbol{\pi}: \boldsymbol{\pi})}[k] z^{-k}$ that are located on the unit circle:

$$
B_{(-\boldsymbol{\pi}: \boldsymbol{\pi})}(z)=\lambda^{2} \sum_{i=0}^{n-1} d_{i}(\boldsymbol{\pi}) z^{-i} \sum_{i=0}^{n-1} d_{i}(\boldsymbol{\pi}) z^{i} .
$$

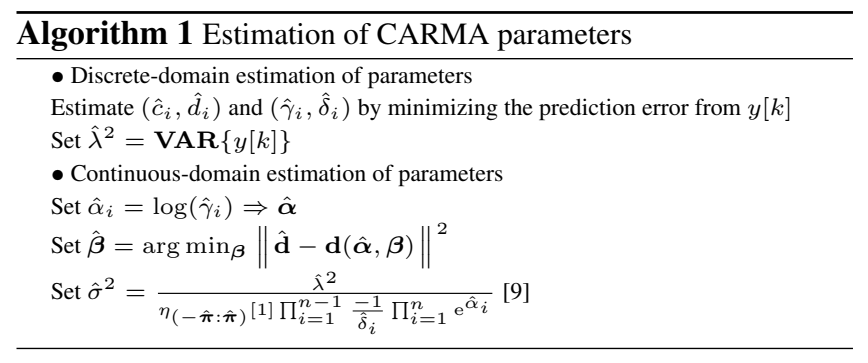

These expressions present a useful link between the discreteand continuous-domain ARMA models, and describe the mapping required to extract CARMA parameters from ARMA coefficients.

\section{CARMA MODEL ESTIMATION}

When the sampling frequency is high enough, the estimation of the CARMA parameters through indirect methods is efficient [8], and there should be no need to consider approaches focusing on several bands [9]. Fortunately, highfrequency sampling operations are prevalent in ultrasound diagnostic. Thus, the derivation of a CARMA model by the inverse mapping of ARMA parameters offers good estimation performances.

We assume here to know the ARMA model orders, which can be selected empirically providing a good fitting of the model to the signal power spectrum. In this selection it is necessary to respect the constraint that the relative order of the discrete-time model must be 1 for the signal to be a sampled CARMA process. The CARMA model order $n$ is constrained to the ARMA orders, while order $m$ can be fixed to provide an equal number of parameters for CARMA and ARMA models.

The ARMA parameters $\left(c_{i}, d_{i}\right)$ and $\left(\gamma_{i}, \delta_{i}\right)$ can be computed by the standard minimization of the prediction error [7], which provides the estimated values $\left(\hat{c}_{i}, \hat{d}_{i}\right)$ and $\left(\hat{\gamma}_{i}, \hat{\delta}_{i}\right)$. While the link between the continuous-domain poles $\alpha_{i}$ and the discrete-domain poles $\gamma_{i}$ is a trivial exponential mapping (in high-frequency sampling conditions), the expression of the discrete-domain zeroes $\delta_{i}$ is a complicated function of both continuous-domain poles and zeroes, as can be seen from (5).

Since $B_{(-\boldsymbol{\pi}: \boldsymbol{\pi})}(z)$ depends solely on the CARMA parameters, it is possible to estimate the zeros $\beta_{i}$ by the numerical minimization of the distance between the estimated ARMA coefficients $\hat{d}_{i}$ and the function $d_{i}(\boldsymbol{\pi})$. The latter can be expressed as $d_{i}(\boldsymbol{\alpha}, \boldsymbol{\beta})$, which highlights the separation between the collection of poles $\boldsymbol{\alpha}$ and the collection of zeroes $\boldsymbol{\beta}$. The parameters $d_{i}(\boldsymbol{\alpha}, \boldsymbol{\beta})$ can be built from the coefficients of the polynomial that corresponds to the roots of $B_{(-\boldsymbol{\pi}: \boldsymbol{\pi})}(z)$ on the unit circle. The collection of coefficients is indicated by d. Likewise, the collection of estimated coefficients of the numerator of the ARMA model numerator is $\hat{\mathbf{d}}$.

Setting $\hat{\lambda}^{2}$ as the variance of the RF signal samples $y[k]$, 
we can estimate $\sigma^{2}$ from the link between the variances of the discrete and continuous innovation signal reported in [9]. The indirect method for CARMA model estimation is summarized in Algorithm 1. We used this algorithm to compute ARMA and CARMA parameters from a sequence of phantom images with various concentrations of scatterers. The two models were compared in terms of classification performance, as described in the next section.

\section{EXPERIMENTS AND RESULTS}

The ultrasound images were realized using as scatterers ultrafine polyamide particles (Orgasol ${ }^{\complement}$, Arkema, France) of diameter $10 \pm 2 \mu \mathrm{m}$ and density $1030 \mathrm{~kg} / \mathrm{m}^{3}$. The tissuemimicking phantoms were prepared by mixing a specific concentration of $\mathrm{Orgasol}^{\circledR}{ }^{\mathfrak{c}}$ particles with distilled water. A magnetic agitator preserved the solution homogeneous throughout the acquisition. In our experiments, we used five different

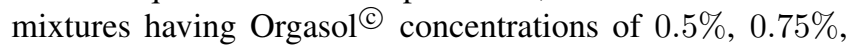
$3 \%, 6 \%$, and $12 \%$. Low and high concentrations mimic random and dense mediums respectively.

The RF signal was acquired by a high-resolution ultrasound scanner (Vevo 770, Visualsonics, Toronto, Canada) at a central frequency of $30 \mathrm{MHz}$ and at a sampling frequency of $500 \mathrm{MHz}$ with 8-bit resolution. For each phantom three video acquisitions were made. A selection of 90 scanlines from the central frame of each video sequence was used (about 300 samples per concentration). Along each scanline, the RF signal was used to compute the ARMA and CARMA parameters, as explained in Section 3, except that we found it is more convenient to numerically optimize real polynomial coefficients, as opposed to complex poles and zeros.

In order to ensure a good fit of the available data, the orders of the CARMA model were chosen as $m=2$ and $n=4$. This provides an $\operatorname{ARMA}(4,3)$ which has been shown a good model for ultrasound signal [3, 4]. Without loss of generality, we constrain the first polynomial coefficients to take a unit value, so that $a_{0}=b_{0}=c_{0}=d_{0}=1$. The process is thus characterized by the four pole parameters $\mathbf{a}=\left(a_{1}, a_{2}, a_{3}, a_{4}\right)$, two zero coefficients, and the variance of the innovation signal, which we represent by the collection $\mathbf{b}=\left(b_{1}, b_{2}, \sigma^{2}\right)$. The orders of the ARMA model are constrained because of the sampling process performed on the continuous-time model: the number $n=4$ of poles is the same, while there are $n-1=3$ zeroes. The ARMA model parameters are represented by the collections $\mathbf{c}=\left(c_{1}, c_{2}, c_{3}, c_{4}\right)$ and $\mathbf{d}=\left(d_{1}, d_{2}, d_{3}\right)$. Finally, the full set of parameters is a seven-attribute vector for both models.

The Mahalanobis distance along a single feature provides information about the individual discrimination power of the considered feature for distinguishing between two concentrations of scatterers [13]. We show in Table 1 the Mahalanobis distance projected onto the direction corresponding to the coefficients on the rows belonging to the two analyzed models.
Table 1. Mahalanobis distance for various concentrations.

\begin{tabular}{llcccc}
\hline \hline Model & & $J_{[.5 \%, .75 \%]}$ & $J_{[.75 \%, 3 \%]}$ & $J_{[3 \%, 6 \%]}$ & $J_{[6 \%, 12 \%]}$ \\
\cline { 3 - 6 } CARMA & $a_{4}$ & $\mathbf{1 4 . 0 1}$ & 10.55 & $\mathbf{2 . 1 3}$ & $\mathbf{4 . 4 4}$ \\
& $b_{2}$ & 11.30 & $\mathbf{3 9 . 1 8}$ & $5 \cdot 10^{-4}$ & 0.17 \\
\hline ARMA & $c_{4}$ & 0.56 & 5.22 & 0.33 & 0.37 \\
& $d_{3}$ & 2.73 & 12.02 & 0.19 & 0.08 \\
\hline \hline
\end{tabular}

We chose the most significant coefficients, separately for each model. The columns of the table represent the binary classification task of two consecutive concentrations of scatterers, where $J_{[x, y]}$ is the Mahalanobis distance between the sample clusters with concentration $x$ and $y$. From this first analysis, it is possible to observe that (i) the higher the concentration of the scatterers, the more difficult the discrimination by a single coefficient; (ii) the CARMA coefficients clearly provide higher distances, which eases the classification task.

To extend these results to the classification of joint parameters, we performed several multiclass discrimination experiments where all available parameters were involved, for both models. The learning scheme used was a support-vector machine with Gaussian kernel, whose parameter had been tuned by cross-validation, separately for each classification model. We report in Table 2 the rate of misclassification (averaged on 20 experiments) for the four multiclass discrimination problems listed in the first column. The classifier was first trained on CARMA and ARMA pole coefficients a and c. Then, it was trained on the zero coefficients and variances $\mathbf{b}$ and $\mathbf{d}$ of both models. Finally, the learning was performed on all the available coefficients for each model.

The classification results show that the proposed CARMA coefficients are more sensitive to differences in the concentration of scatterers, above all when the discrimination is multiclass. Continuous-domain parameters favour a better recognition score and represent tissues of different concentrations in more compact and better discernible clusters, as illustrated in Figure 1. The seven-dimensional samples in these scatter plots are normalized and projected onto a plane where they have, at the same time, maximum inter-class distance and minimum intra-class variance [13]. The bivariate distribution of samples is described by a 2D Gaussian fitting for each category, whose ellipse at a probability density function of $80 \%$ is shown in the figures. We observe that the clusters are better defined in the space of CARMA coefficients than in the space of the traditional ARMA parameters.

The difference of performances between CARMA-based and ARMA-based classifiers of the last two columns in Table 2 is also verified by resampled paired t-tests. These statistic tests, performed on the misclassification errors obtained in 20 experiments, indicate in all the four classification problems an increment in performance of CARMA-based classifier respect to ARMA-based classifier at a $5 \%$ significance level. The improvement of performance is significant and encourages the application of continuous-time models on in vivo images for the characterization of ultrasound tissues. 
Table 2. Rate of misclassification: [mean value \pm standard deviation] of 20 experiments $\left({ }^{1}\right.$ CARMA model, ${ }^{2}$ ARMA model $)$.

\begin{tabular}{|c|c|c|c|c|c|c|}
\hline \multirow{3}{*}{$\begin{array}{l}\text { Classes } \\
\text { Concentration }\end{array}$} & \multicolumn{6}{|c|}{ Features for classification } \\
\hline & \multicolumn{2}{|c|}{ poles } & \multicolumn{2}{|c|}{ zeroes } & \multicolumn{2}{|c|}{ all parameters } \\
\hline & $\mathbf{a}^{1}$ & $\mathbf{c}^{2}$ & $\mathbf{b}^{1}$ & $\mathrm{~d}^{2}$ & $(\mathbf{a}, \mathbf{b})^{1}$ & $(\mathbf{c}, \mathbf{d})^{2}$ \\
\hline$[0.5 \%, 0.75 \%]$ & $0.007 \pm 0.005$ & $0.024 \pm 0.006$ & $0.013 \pm 0.005$ & $0.008 \pm 0.003$ & $0.007 \pm 0.003$ & $0.010 \pm 0.003$ \\
\hline$[0.5 \%, 0.75 \%, 3 \%]$ & $0.010 \pm 0.003$ & $0.024 \pm 0.004$ & $0.008 \pm 0.003$ & $0.032 \pm 0.006$ & $\mathbf{0 . 0 0 3} \pm 0.002$ & $0.012 \pm 0.003$ \\
\hline$[0.5 \%, 0.75 \%, 3 \%, 6 \%]$ & $0.116 \pm 0.006$ & $0.127 \pm 0.006$ & $0.156 \pm 0.007$ & $0.270 \pm 0.008$ & $\mathbf{0 . 0 6 9} \pm 0.006$ & $0.113 \pm 0.006$ \\
\hline$[0.5 \%, 0.75 \%, 3 \%, 6 \%, 12 \%]$ & $0.126 \pm 0.007$ & $0.135 \pm 0.006$ & $0.273 \pm 0.009$ & $0.368 \pm 0.010$ & $\mathbf{0 . 0 8 5} \pm 0.006$ & $0.122 \pm 0.006$ \\
\hline
\end{tabular}

\section{CONCLUSIONS}

We presented a continuous-time ARMA model for RF ultrasound signals and proposed an indirect approach to compute CARMA parameters based on the properties of exponential B-splines. This work includes a comparative study of the proposed CARMA coefficients and of the traditional ARMA parameters that are widely used for the analysis of ultrasound images. We performed experiments on phantom images specifically designed with various concentrations of scatterers. We focused on the comparison of CARMA and ARMA features in terms of discrimination performances. The experimental results reveal that the new CARMA features are able to capture more efficiently the information about the concentration of scatterers. Thus, it can be extremely useful for the characterization of in vivo ultrasound tissues.

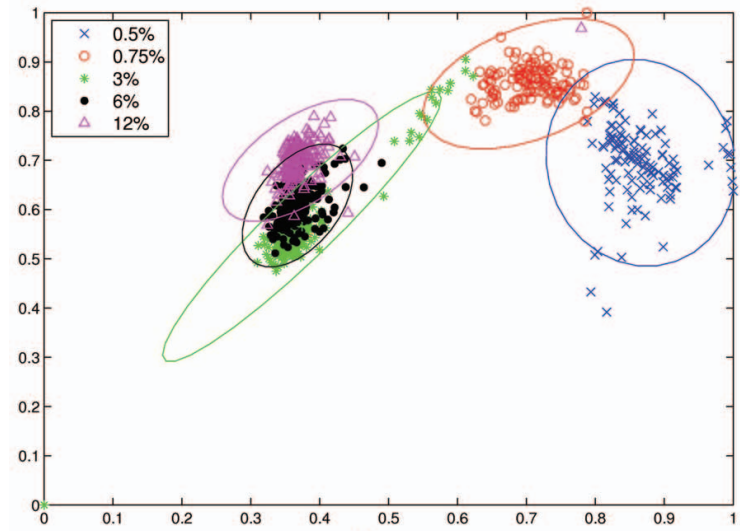

(a)

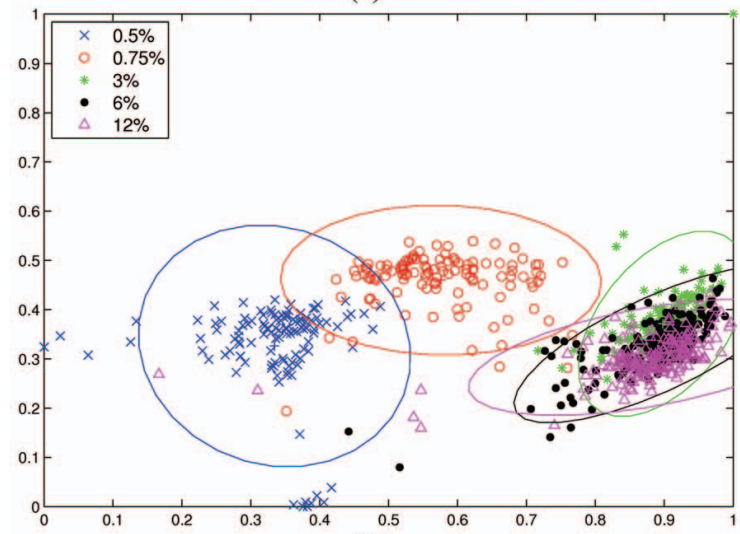

(b)

Fig. 1. Scatter plots of normalized CARMA parameters in (a) and ARMA coefficients in (b) for several scatterer concentrations.

\section{REFERENCES}

[1] O.V. Michailovich and D.R. Adam, "Deconvolution of medical images from microscopic to whole body images," in Blind Image Deconvolution: Theory and Applications, P. Campisi and K. Egiazarian, Eds., chapter 5, pp. 169-230. CRC Press, 2007.

[2] S. Maggio, A. Palladini, L. De Marchi, M. Alessandrini, N. Speciale, and G. Masetti, "Predictive deconvolution and hybrid feature selection for computer-aided detection of prostate cancer," IEEE Trans. Med. Imag., vol. 29, no. 2, pp. 455-464, February 2010.

[3] A. Abdulsadda, N. Bouaynaya, and K. Iqbal, "Stability analysis and breast tumor classification from 2D ARMA models of ultrasound images," in Proc. 31th Annual Int. Conf. IEEE Eng. in Med. and Biol. Soc. (EMBS'09), Minneapolis MN, USA, September 3-6, 2009, pp. 3763-3766.

[4] J. Zielinski, N. Bouaynaya, and D. Schonfeld, "Twodimensional ARMA modeling for breast cancer detection and classification," in Proc. of the 2010 IEEE Int. Conf. Signal Process. and Commun. (SPCOM'10), Bangalore, India, July 18-21, 2010, pp. 1-4.

[5] H. Kirshner and M. Porat, "On the role of exponential splines in image interpolation," IEEE Trans. Image Process., vol. 18, no. 10, pp. 2198-2208, October 2009.

[6] S. Nemirovsky and M Porat, "On texture and image interpolation using Markov models," Signal Process.: Image Commun., vol. 24, no. 3, pp. 139-157, March 2009.

[7] L. Ljung, Ed., System Identification: Theory for the User, Information and System Sciences. Prentice Hall, 2 edition, 1999, $672 \mathrm{p}$.

[8] H. Garnier and L. Wang, Eds., Identification of ContinuousTime Models from Sampled Data, Advances in Ind. Control. Springer, 2 edition, 2008, 413 p.

[9] S. Maggio, H. Kirshner, and M. Unser, "Continuous-time AR model identification: Does sampling rate really matter?," in Proc. 18th European Signal Process. Conf. (EUSIPCO'10), Ålborg, Denmark, August 23-27, 2010, pp. 1469-1473.

[10] M. Unser and T. Blu, "Cardinal exponential splines: Part ITheory and filtering algorithms," IEEE Trans. Signal Process., vol. 53, no. 4, pp. 1425-1438, April 2005.

[11] M. Unser, "Cardinal exponential splines: Part II-Think analog, act digital," IEEE Trans. Signal Process., vol. 53, no. 4, pp. 1439-1449, April 2005.

[12] O.V. Michailovich and D. Adam, "A novel approach to the 2D blind deconvolution problem in medical ultrasound," IEEE Trans. Med. Imag., vol. 24, no. 1, pp. 86-104, January 2005.

[13] A.R. Webb, Ed., Statistical Pattern Recognition, Wiley, 2 edition, 2002. 